\title{
PAISAJES, VIDAS Y EQUIVOCACIONES EN LOS ANDES MERIDIONALES (JUJUY, ARGENTINA) ${ }^{1}$
}

\author{
LANDSCAPES, LIVES AND EQUIVOCATIONS IN SOUTHERN ANDES \\ (JUJUY, ARGENTINA)
}

\author{
Francisco Pazzarelli ${ }^{2}$ y Verónica S. Lema ${ }^{3}$
}

\begin{abstract}
En este trabajo presentamos algunas reflexiones que ponen a la noción de paisaje en variación, considerando las formas equívocas (sensu Viveiros de Castro 2004), desde las cuales puede ser pensada y constituida. A partir de nuestras experiencias de campo en la comunidad aborigen de Huachichocana (Jujuy, Argentina) reflexionamos sobre la posibilidad de habitar mundos múltiples, controlando equivocaciones sin pretender erosionar las diferencias, ni reducir los conflictos a procesos de hibridez o mezclas. Procuramos acompañar las reflexiones de nuestros interlocutores y la posibilidad de pensar otros acercamiento posibles a la idea de paisaje, que nos permita sortear las contradicciones modernas que proponen relaciones del tipo: paisaje 'inerte' vs. paisaje 'vivo'. Consideramos que las relaciones de algunos de estos paisajes se presentan como irreductibles a 'un' mundo y reclaman más de uno para ser comprendidas. Finalmente, sugerimos que estas deben ser pensadas bajo la forma de conexiones parciales.

Palabras claves: paisaje, equivocación, diferencia, Andes.
\end{abstract}

In this paper, we present some reflections on alternative interpretations of the notion of landscape based on the equivocal ways (sensu Viveiros de Castro 2004) in which it can be thought of and constituted. From our fieldwork experience in an Aboriginal community in the province of Jujuy, we consider the possibility of inhabiting multiple worlds by controlling equivocations, attempting neither to erode their differences nor to reduce conflicts to processes of hybridity or mixing. We seek to accompany the reflections of our interlocutors and to advance towards the possibility of thinking of other possible approaches to the idea of landscape, which can allow us to overcome the modern contradictions that propose binary relations and options, such as 'inert' landscape vs. 'living' landscape. We believe that the relations of some of these landscapes are irreducible to 'one' world, as they require more than one to be understood. Finally, we suggest that these connections should be thought of as partial connections.

Key words: Landscape, equivocation, difference, Andes.

\section{Paisajes}

En innumerables ocasiones, al sacar fotos junto con nuestros amigos de Huachichocana (Comunidad Aborigen emplazada en Jujuy, Argentina) ellos nos recomiendan ciertas perspectivas: que se vea tal o cual montaña, alguna casa, un cardón de formas exuberantes, las siluetas de las cuevas a lo lejos, los antiguos caminos, el verdear de las faldas del cerro en el verano. Muchas veces fueron ellos quienes, cámara en mano, tomaron las instantáneas que luego ilustraron algunos de los textos que escribimos conjuntamente (Cruz de Tolaba et al. 2015), buscando tomas especiales para recordar algo en particular o la belleza en una foto con el paisaje de los cerros de fondo ${ }^{1}$. En principio, nadie se ve extrañado por expresiones o actitudes de ese tipo y mucho menos por usarlas frente a alguno de los turistas que se acerca a realizar caminatas o visitar los sitios arqueológicos de la región. Sin embargo,

\footnotetext{
1 Una primera versión de este trabajo fue presentada en la VIII Reunión de Teoría Arqueológica de América del Sur (TAAS), en el Simposio "Paisajes en Conflicto", co-organizado junto a Eduardo Herrera Malatesta, realizado en La Paz, Bolivia, mayo de 2016. Este manuscrito fue evaluado por pares externos y editado por el Comité Editorial de Chungara y Dante Angelo en su calidad de editor invitado.

2 Instituto de Antropología de Córdoba-CONICET, Universidad Nacional de Córdoba, Córdoba, Argentina. fpazzarelli@hotmail.com

3 Instituto de Humanidades-CONICET, Universidad Nacional de Córdoba, Córdoba, Argentina. vslema@ gmail.com
}

Recibido: marzo 2017. Aceptado: febrero 2018.

http://dx.doi.org/10.4067/S0717-73562018005000602. Publicado en línea: 6-abril-2018. 
ello tampoco supone que todas las relaciones que componen ese paisaje se suturen en alguna noción de background natural sobre el cual se desarrollan las historias humanas, como lo sería desde la perspectiva de un "paisaje moderno". Con este punto de partida, entonces, en este trabajo reflexionaremos sobre algunas de estas relaciones retomando un clásico problema de buena parte de la etnología regional: el de la ambigüedad constitutiva, no contradictoria, de las montañas, cerros y otros seres del 'paisaje andino'. Estas discusiones, que fueran planteadas por Martínez (1983) hace ya varias décadas son la inspiración para considerar aquí ciertas articulaciones del paisaje huacheño donde, por ejemplo, los cerros pueden aparecer como el 'fondo' de una fotografía, pero también 'vivir' en el marco de relaciones específicas a las ontologías andinas.

Huachichocana es una pequeña comunidad aborigen que pertenece al departamento Tumbaya, en la provincia de Jujuy, donde trabajamos con distintos proyectos de investigación desde $2010^{2}$. Está compuesta por familias que se dedican al pastoreo de cabras y ovejas (con algunas llamas y vacas), animales que conforman la hacienda a partir de la cual producen y venden quesos. También desarrollan una actividad agrícola dedicada principalmente a la papa, maíz y alfalfa. Las familias poseen casas y puestos (residencias estacionales) en distintos parajes de los pisos puneños y prepuneños, por los que circulan a lo largo del año siguiendo a la hacienda y el curso de las estaciones y lluvias. En el amplio territorio que alberga a estos movimientos, se encuentran sitios arqueológicos y estaciones de arte rupestre. Desde una perspectiva regional, Huachichocana presenta muchas resonancias con etnografías realizadas en los Andes meridionales.

Existen muchos trabajos dedicados a analizar los dislocamientos entre formas modernas-occidentales de percibir al paisaje y aquellas otras (no modernas, indígenas) que reconocen un conjunto de seres y relaciones allí donde otros solo ven espacios naturales. Incluso podríamos afirmar que la mayoría de las etnografías en la región andina posee algún tipo de reflexión sobre el tema; nos interesan especialmente aquí aquellas dedicadas al área meridional (Allen 2002; Arnold et al. 1992; Bouysse-cassagne y Harris 1987; Cruz 2012; Gose 2001; Martínez 1989; Spedding 1992; Spedding y Arnold 2009) que incluye a las tierras del noroeste argentino (Bugallo 2008; Bugallo y Tomasi 2012; Bugallo y Vilca 2011; Haber 2007; Tomasi 2011; Vilca 2009, 2012). Desde distintas perspectivas, estas investigaciones presentan siempre algún tipo de crítica al "paisaje", tal y como lo definiría un esquema moderno de pensamiento. Estos esquemas pueden ser presentados en resonancia con la forma en que fueran definidos por Latour (1991), un concepto de "paisaje" que reduce las formas de relación con lo natural a esquemas de oposición con algo que sería estrictamente humano; esto es, una concepción de la "naturaleza" como un compartimiento diferente (y un recurso a disposición) de la sociedad y la cultura. Se discute la posibilidad de que una idea así pueda ser utilizada acríticamente para abordar nociones nativas sobre el paisaje o la naturaleza, algo que en última instancia develaría una operación colonialista y hegemónica sobre el pensamiento ajeno. En otras palabras, se argumenta que ese paisaje moderno no toleraría la diferencia andina: no consigue albergar en su interior a los incontables casos etnográficos y arqueológicos que constantemente desafían sus premisas (ver también Gow $\left.1995^{3}\right)$.

A tono con estas discusiones, en los últimos años escribimos diferentes trabajos que se ocupaban de temas vinculados a diferentes dimensiones del paisaje y a su ritualidad asociada. Lo hicimos teniendo en cuenta las formas nativas en que estas relaciones se presentaban y en todos los casos nuestras discusiones siempre terminaban con alguna referencia a los "paisajes vivos" de los Andes, incorporando las críticas y comentarios de los autores mencionados. En este trabajo, nos proponemos retornar a ellos de modos más explícitos para colocarlos en variación, considerando las formas equívocas (sensu Viveiros de Castro 2004) desde las cuales pueden ser abordados. Para ello sugerimos pensar a los "paisajes vivos" como agenciando la posibilidad de habitar mundos múltiples, con conexiones parciales (Strathern 2004) que no se reducen a procesos de hibridez, mezclas u oposiciones simples (paisajes inertes vs. paisajes vivos). En las secciones siguientes ahondaremos en estas ideas a través de descripciones y reflexiones sobre antigales y humos; en ambos casos sugerimos que muchas relaciones se presentan como irreductibles a 'un' tipo de mundo. Finalizaremos con una reflexión sobre los modos equívocos y productivos que definen buena parte de estas situaciones y que existen también al interior de las lógicas locales.

\section{Paisajes Vivos}

Podríamos decir que en Huachichocana, al igual que en otras comunidades de los Andes, las relaciones con cerros, lagunas, plantas, animales o sitios arqueológicos, no pueden ser suturadas en una idea moderna de paisaje: relaciones con plantas y animales domesticados y silvestres, memorias, territorio y sitios arqueológicos, vínculos con cerros, ojos de agua y lagunas. Fue en esa dirección 
que algunos de nuestros trabajos se ocuparon de discutir la 'naturaleza' local (Lema 2013, 2014a, 2014b; Lema y Pazzarelli 2015; Pazzarelli 2014, 2016a, 2016b, 2017) permitiéndonos sugerir, en consonancia con otros autores, que el paisaje huacheño está "vivificado" o "vivo" (Spedding 1992; Vilca 2009). Una idea así invierte la concepción moderna: el paisaje ya no está inerte como un objeto, como un fondo inanimado, sino poblado de seres y fuerzas; está vivo como un sujeto. A partir de allí, se abre un nuevo campo para la descripción y abordaje de las naturalezas andinas.

No obstante, también es cierto que los huacheños tejen y se involucran cotidianamente en relaciones con distintos tipos de mundos. Eso hace que en ciertas ocasiones entidades potentes como los cerros pueden ir de fondo en la foto de un turista, las relaciones con las plantas parecen ser discutidas como recursos al hablar con técnicos, la tierra puede devenir objeto de propiedad individual transferible y los antigales (espacios habitados en el pasado, reconocidos por su potencia y peligrosidad) pueden ser presentados como sitios arqueológicos de miles de años de antiguedad en un libro escrito colectivamente o incluso manejados turísticamente (Lema y Pazzarelli 2018; ver también De la Cadena 2015; Spedding y Arnold 2009). ¿Dónde quedaría la vida del paisaje y de sus seres en todos esos casos (comunes, además, a otras comunidades andinas)?

Para algunos analistas, la simultaneidad de estas relaciones (la tierra como ser potente y objeto de propiedad catastral) sería una confirmación de que la "vida" del paisaje no es más que una creencia, un esencialismo cultural que puede eventualmente suspenderse o desaparecer. Para otros, las relaciones que las comunidades indígenas traban con el Estado, sus agentes (técnicos, ministerios) u otros (turistas, antropólogos) son la mayoría de las veces estratégicas e instrumentales. En estos casos, la vida de los seres del paisaje podría ser ocultada o silenciada en situaciones poco favorables; las comunidades se presentarían ante los demás tal y como estos desean verlas, manipulando estratégicamente su 'cultura' y mostrando solo las partes más palatables para una audiencia occidental. En estos contextos, algunos pensarían que la tarea del antropólogo sería justamente separar aquello que cierta performance indígena habría mezclado, mostrando que las comunidades dicen lo que el Estado desea oír, pero secretamente piensan y sienten otra cosa. Una postura así nos llevaría, por un lado, a definiciones del paisaje y la naturaleza andina demasiado afincadas en ideas de mestizajes y sincretismos, bastante rígidas y de pura naturaleza utilitaria y estratégica (como lo discutieron algunas autoras,
Bouysse-Cassagne 2004; Rivera Cusicanqui 2010). Pero más importante, nos alejaría de cualquier tipo de simetría en las relaciones abordadas, pues finalmente en estas situaciones ¿cuál es el lugar de los seres y fuerzas del paisaje? ¿Aparecen solo como objetos pasivos de manipulaciones y estrategias? ¿O acaso los cerros tendrían algo para decir o hacer al respecto?

Entendemos que no existe una salida fácil para estas preguntas. Afirmar que el paisaje está "vivo" invierte las posiciones modernas de sujeto-objeto, es verdad; y esta inversión es metodológicamente indispensable para que cierta 'vida' pueda emerger. Pero no es suficiente como respuesta final, desde el momento en que mantiene una relación entre dos términos donde no caben muchas de las experiencias huacheñas con otros, con el mundo moderno (en el sentido latouriano). Spedding y Arnold (2009:315), por ejemplo, reclaman considerar la posibilidad de que la fuerza de algunos ritos asociados a la geografía sagrada del altiplano boliviano se despliega para hacer crecer los negocios locales conectados con las economías globalizadas. De algún modo, se trataría de pensar a los cerros como ayudando a ganar dinero. En el mismo sentido, Arnold (2009) se pregunta por la posibilidad de una teoría etnográfica que se ocupe de los modos de relación de los pueblos andinos con los sistemas económicos a los que pertenecen desde hace siglos, reclamando una perspectiva que valorice las formas nativas de pensar estas interacciones (que no imitan a las lógicas occidentales sino que se fundan, entre otras cosas, en nociones particulares de humanidad y cuerpo). Es decir, ambas autoras reclaman una mirada que no esencialice el estudio del pensamiento andino sobre el espacio y el paisaje (definiéndolo como naturalmente incapaz de conectarse con otros), pero tampoco lo desestime al momento de pensar los vínculos de los pueblos indígenas con el mundo moderno, el Estado y sus resistencias (como si la creatividad del pensamiento indígena hubiera sido barrida de las montañas). En otras palabras ¿sería posible pensar simétricamente en relaciones con el paisaje que pueden conectarse, casi simultáneamente (y en ocasiones solo momentáneamente), con el 'mundo andino' y el 'mundo moderno'?

Para avanzar sobre este punto, creemos que sería necesario revisar primero los modos en que se definen estas relaciones. Si nuestro primer paso hasta aquí fue acompañar la idea de un "paisaje vivo", lo que sigue ahora es asumir que esa "vida" no puede ser abordada en los términos de una noción abstracta o trascendental. Sostenemos que 'vida' es otra forma de decir 'relación'; y las relaciones pueden ser cosas diferentes de las que pensamos (Strathern 2004). En otras palabras, poner en 
variación el concepto de paisaje considerando las formas equívocas y conflictivas que lo constituyen, abordando simétricamente a los múltiples modos (y no solo algunos) en que nuestros interlocutores piensan sobre y con la vida de los cerros. En definitiva, se trata menos de dar una definición convincente de lo que el paisaje es o no es, y más bien ocuparnos de atender a los modos locales y "equivocaciones" que complican el camino hacia esa definición.

\section{Equivocaciones}

Hablar de equivocaciones nos lleva a explicitar algunos recaudos metodológicos que aprendimos en nuestro trabajo de campo. En primer lugar, que preguntarnos por el paisaje (y otras cosas) en Huachichocana era primero reconocer que debíamos aprender aquellos otros idiomas que se expresaban en nuestra propia lengua y que muchas veces prescindían de comunicaciones verbales (FavretSaada 1990). Cuando un huacheño nos señalaba un cerro particular durante una caminata o nos explicaba su nombre e historia, esos gestos y palabras contenían potencialmente todas las relaciones que los implicados en la conversación podían reclamar.El paisaje o "cerro" enunciado en el castellano andino de la región, mostrado por un huacheño al tomar una fotografía, recomendar un mejor ángulo o hacer alguna interpretación, no era completamente nuestro paisaje, pero estaba conectado con él. Sobre esta capacidad huacheña para agenciar y conectarse potencialmente con múltiples diferencias presentaremos algunos ejemplos en lo que sigue; "conexiones parciales" que se producen cuando diferentes puntos de vistas se conectan bajo la forma de una relación que es más que una, pero menos que dos (Strathern 2004). Estas conexiones parciales se encuentran implicadas metodológicamente bajo lo que Viveiros de Castro denominó "equivocación". Una equivocación, antes que un simple error o falla en la comunicación es "una falta de comprensión de que los entendimientos no son necesariamente los mismos y que no están relacionados a formas imaginarias de 'ver el mundo', sino a los mundos reales que están siendo vistos" (Viveiros de Castro 2004:11 -nuestra traducción). Conexiones entre formas homónimas de referir a las 'mismas' relaciones que, sin embargo, se abren hacia mundos diferentes.

Pensar en conexiones parciales no solo pone en variación las ideas de encuentros y mezclas, sino que también habilita a pensar a ciertos paisajes y seres con la posibilidad de habitar entre mundos, sin por ello tornarse indefinidas o caricatura de otras cosas (De la Cadena 2015). De algún modo, se trata de volver a pensar el encuentro (de relaciones, seres, fuerzas, conceptos) tal y como sugiere la noción de ch'ixi para el área andina (Rivera Cusicanqui 2010): una convivencia intensiva de diferencias que no se reduce de antemano a una fusión, hibridez o a un simple proceso sincrético resuelto en la generación de un tercer elemento ${ }^{4}$. La posibilidad de un 'paisaje vivo', entonces, se conecta con la necesidad de considerar a esa 'vida' como reclamando un tipo de relacionalidad específica. No bajo la forma de una vitalidad trascendental, sino de agenciamientos particulares que hacen emerger la vida de cerros y personas.

En este sentido, el cerro (las personas y cualquier otro ser) siempre están vivos 'para' alguien, habitando conexiones parciales. En lo que sigue, sugeriremos entonces que estas conexiones se actualizan bajo la forma de relaciones mutuas que ayudan a "sostener" los lugares (Martínez 1989). Todo se encuentra imbricado en relaciones donde las cosas deben 'llegar a ser junto con otras' en el marco de implicaciones "mutuas" y "forzosas" (Martínez 1989), caracterizadas por la literatura regional como "crianzas mutuas" (Bugallo y Tomasi 2012; Haber 2007; Lema 2013, 2014a, 2014b). Discutiremos esto considerando dos conjuntos de equivocaciones: aquellas manifestadas en torno de los sitios arqueológicos al momento de abordar la historia local y las observadas durante un proyecto de desarrollo vinculados a cocinas.

\section{Antigales}

Algunos sitios arqueológicos son llamados de antigales, pues allí vivían los antiguos. Aunque no se reconoce una filiación directa entre los antiguos y los huacheños actuales, estos sitios son especialmente considerados como lugares potentes del paisaje. Junto con lagunas, cerros, ojos de agua, cuevas, rocas, ríos, rastrojos, corrales, antiguas explotaciones mineras y casas abandonadas, encarnan el paisaje "vivo" de Huachichocana. Algunos de estos lugares (los antigales o las explotaciones mineras) podrían ser considerados desde una mirada foránea como menos 'naturales' que otros. Sin embargo, todos están allí y junto con las personas dan forma a la red de "crianzas mutuas" y a la memoria del lugar. Fueron estas relaciones las que desataron el primero de los nudos de la equivocación sobre el que queremos reflexionar en este trabajo: aquel que nos involucró cuando intentamos reconstruir la historia de la comunidad en un libro, pues cada vez que preguntábamos por el 'pasado' los huacheños nos llevaban a recorrer sus lugares 5 .

En un trabajo previo abordamos la relación entre memoria, crianza y los vínculos de actualizaciones entre ambas (Lema y Pazzarelli 2015). Si volvemos 
a las crianzas mutuas aquí, es porque ellas encarnan todo lo que de relevante tienen las relaciones locales con el paisaje, manifestándose especialmente bajo la forma de diferentes tipos de ofrendas de comidas. Los rituales de dar de comer que acontecen en agosto, por ejemplo, se encuentran destinados a ofrecer comidas de todo tipo a Pachamama, entre ellas preparaciones especiales como la tijtinch $a^{6}$, bebidas, coca, cigarros y dulces. Las bocas de Pachamama se encuentran definidas material y espacialmente en diferentes lugares del paisaje habitado: dentro de las habitaciones de las casas, en los patios, corrales y jardines, en rastrojos y sembraderos, en ojos de agua, canales y zonas de pastoreo. Cada uno de estos lugares es recorrido y alimentado. Muchos son también cotidianamente challados: las challas pueden incluir una pequeña libación (desde vino y licores hasta alcohol medicinal), el ofrecimiento de cigarrillos o de hojas de coca fresca. Generalmente, las personas acompañan el gesto bebiendo un trago, fumando o coqueando. Además, se challan lagunas, ciertos tramos de caminos, algunas rocas especiales, cuevas y apachetas (montículos de piedra). En algunos lugares particulares, vinculados a las circulaciones de larga distancia (cuevas, rocas importantes, paredones, apachetas, antigales), las challas pueden incluir achos, bolos mascados de coca (Pazzarelli y Lema 2015). En realidad, cualquier espacio del paisaje local puede ser potencialmente challado y a nadie le sorprendería que un lugar que nunca fue objeto de atenciones rituales comience a serlo en algún momento en particular (ver p. ej. Spedding y Arnold 2009). Todo depende de la configuración actual de la red de crianzas. Como cuando los huacheños nos señalaron a la piedra winchi, que inicialmente pensamos se trataba de alguna toponimia de origen quechua (que abundan en la región), pero luego nos enteramos que refería al parecido de la roca con el mango de un rifle Winchester, que a su vez recordaba el gusto por la caza de unos familiares antiguos ${ }^{7}$.

Aquello que hace que esta constelación de seres del paisaje (que incluye a la piedra winchi) se encuentre ‘vivo' es su articulación con distintas prácticas ritualesalimenticias que posibilitan que ellos también críen. Ambas formas, el dar de comer y la challa, revelan a la ingesta y a la comensalidad como formas privilegiada de relación con 'otros', estableciendo vínculos de crianza y habilitando un lugar en la memoria local. De este tipo de relaciones depende la fertilidad de aquello que está en juego (agua, cultivos, animales, circulación), una responsabilidad que recae tanto en quienes alimentan o challan, como en los seres alimentados que devuelven a las personas aquello que están necesitando. Todo ello sin perder su peligrosidad y su potencial de enfermar y devorar a animales y personas desprevenidos o maleducados, que no respeten los códigos de estas interacciones. En resumen, esta red de crianzas incorpora entre sus términos a todos aquellos seres y relaciones implicados con el desarrollo de la vida y fertilidad actual, es decir, con las formas que las relaciones de crianzas adoptan en el presente. Esto supone un desdoblamiento importante y no siempre considerado en la literatura: aunque el paisaje huacheño se encuentre marcado de diferentes formas y como consecuencia de distintos eventos históricos, eso no significa que todos ellos sean materia prima para el recuerdo o las atenciones rituales. Lo serán solo aquellos que actualmente ayudan a sostener-criar los lugares (cf. Martínez 1989).

Entre esos lugares-seres se encuentran las cuevas de Huachichocana, que poseen tanto vestigios arqueológicos como paneles de arte rupestre. Las cuevas (particularmente algunas), el cerro que las contiene y el estrechamiento de la quebrada donde se emplazan, constituyen un punto potente del paisaje donde se challa al caminar de abajo hacia arriba, se ofrenda al pachero (una pequeña boca donde se liba) que existe dentro de la cueva grande, o se arrojan achos contra las paredes. Se trata de un lugar particularmente peligroso que atrae, puede devorar a los codiciosos y donde incluso se producen encuentros con otros seres. Esto ocurre también en otros espacios con arte rupestre. Lo interesante, es que esto no sucede con todos: algunos de estos sitios arqueológicos han sido 'olvidados', no solo porque actualmente son espacios prácticamente deshabitados, sino porque han quedado por fuera de las redes de crianza, desactivados (como los destacados paneles rupestres de Tocoleras, una de las quebradas del lugar). Otros, en cambio, aunque también lejanos de donde vive la gente actualmente siguen siendo considerados antigales potentes y frecuentemente challados. Es decir, algunos sitios puedan ser reconocidos como milenarios y potentes criadores, mientras otros son completamente desconsiderados (aunque para la arqueología todos sean milenarios e importantes).

Lo anterior nos permitió sugerir que la memoria local (y su código culinario) se presta solo a aquellos que crían cada día el lugar (Lema y Pazzarelli 2015). Se trata así de una memoria vinculada a un presente criador, que no tiene necesariamente que ver, en principio, ni con una historia, ni con un pasado; o al menos no con nuestra historia llena de progresiones y secuencias. Solo quienes crían constituyen materia prima para el recuerdo y como la de criador es una condición que se gana ejerciéndola, no 
existe posibilidad lógica de criadores por fuera del espacio práctico, pues todos ellos deben ser 'activos' (y de nada sirve apelar a una ancestralidad trascendental si las relaciones que la definen no se encuentran activadas en el presente, dando de comer o challando $)^{8}$. La renovación diaria y ritual de los pactos entre personas y otros lugares/seres es lo que permite seguir formando parte de la red de criadores y de su memoria (ver también Molinié 1997).

Cuando comenzamos nuestras indagaciones sobre el pasado del lugar, entendíamos que el paisaje y sus espacios serían parte importante de la narración. No imaginábamos, sin embargo, que challar y dar de comer eran requisitos indispensables para activar la memoria de una historia que era puro presente. Creíamos estar hablando de lo 'mismo', pero la 'historia huacheña' resultó ser la memoria presente y fértil de un paisaje con hambre. Hablar sobre 'historia' suponía un campo relacional abierto hacia el mundo del paisaje 'vivo' y de su memoria fértil; desde allí (y no antes) se nutría de la línea cronológica de las familias que poblaron el lugar, junto a otros 'hitos históricos' como el emplazamiento de la escuela primaria local en diferentes casas de la comunidad o el trazado de una ruta nacional -o la piedra winchi! (Lema y Pazzarelli 2015) ${ }^{9}$. Todo esto no podía dejar de afectar la práctica arqueológica.

A diferencia de otras comunidades cercanas, los huacheños han sido menos recelosos con la excavación del interior de las cuevas, o con la manipulación de los restos obtenidos ${ }^{10}$. Su recelo (con nuestro trabajo pero también, según sus relatos, con el de los arqueólogos que antes trabajaron allí) se manifiesta en relación a nuestros vínculos (casi personales) con las cuevas, antigales y otros sitios potentes. Se interesan por saber si realizamos las challas que corresponden $\mathrm{y}$, especialmente, si las hacemos con fe. En este sentido, ellos modulan nuestra práctica, que no empieza ni termina con cada campaña de excavación. Ella se extiende a momentos como el mes de agosto, cuando la tierra está abierta, hambrienta, particularmente peligrosa y no se puede excavar. En los eventos de dar de comer de agosto se nos enfatiza que debemos hacerlo bien y confe, pues trabajamos con antigales. Se nos señala que estos lugares potentes pueden enviciarnos, suelen tentar y generar avaricia, provocando enfermedades o, en el peor de los casos, devorando a los intrusos ${ }^{11}$. Excavar conecta, así, al menos dos protocolos: la metodología arqueológica y los códigos de comportamiento con un cerro, cueva y antigal vivo, potente y peligroso. Todo esto sin que la especificidad de cada uno se vea anulada por la del otro. Los huacheños muestran que la cueva solo puede ser intervenida si ella acepta nuestras challas; una negativa sería rápidamente identificada bajo la forma de enfermedad. En otras palabras, la equivocación entre arqueólogos y huacheños tiene una tercera parte, la cueva, que también se involucra en la relación y puede decidir no aceptar esa conexión. Tal como cuando se abre la tierra para alimentarla y se inspeccionan los huesos de la tijtincha del año anterior para evaluar si la tierra recibió bien (aceptó la ofrenda).

En otras palabras, la comprensión huacheña del antigal potente, fértil y criador no cancela a priori la posibilidad de que sea excavado y potencialmente presentado como un sitio arqueológico a los turistas. En el mismo sentido, se nos reclama de nuestro saber arqueológico para que ellos puedan contar esa 'otra historia' a los turistas, una que se alinea con la historia cronológica de las familias de la comunidad, de las escuelas, minas y rutas. Esa historia cronológica y lineal es tan huacheña como la memoria fértil, pues la primera está pensada a partir de la segunda y así conectadas; allí reposa el antecedente que permite pensar en proyectos de turismo 'bien hechos' como fuentes potenciales de articulación con entidades estatales y privadas, que incluyan ingresos $\operatorname{monetarios}^{12}$. En estos casos, quizá los huacheños están pensando en el Estado y sus exigencias (leyes de patrimonio, protocolos de autorización para excavaciones, reglamentación del turismo y de museos), procurando conectar en términos fértiles que abreven en el sostenimiento local, teniendo que explorar (e inestablemente controlar) los potenciales equívocos. En esa red entramos los arqueólogos, involucrados en los flujos de crianza cuando excavamos y challamos al pachero dentro de la cueva. Relaciones similares pueden encontrarse en otros proyectos como el que analizaremos a continuación.

\section{Humos}

Hace algunos años, en el marco de proyectos del Programa de las Naciones Unidas para el Desarrollo (PNUD), llegaron a la comunidad proyectos que proponían la instalación de cocinas económicas para regular algunos problemas vinculados a las prácticas locales. De acuerdo a la información provista por estas instituciones (PNUD 2009) y en entrevistas con los técnicos a cargo, estos problemas se resumían en dos: el uso desproporcionado de leñas (asociado con procesos de desertificación en la región) y las emisiones excesiva de humos y gases $^{13}$. Estas cocinas, ahora ya instaladas, adoptan diferentes formas, pero en todos los casos suponían remplazar a los fogones tradicionales por estructuras construidas con adobes, chapas y chimeneas (se 
articulaban también con hornos económicos, aunque no nos ocuparemos de ellos aquí). En ellas, las leñas se introducen en espacios cerrados a la manera de cajas que permiten regular la entrada de aire y el proceso de combustión, extrayendo el humo fuera del cuarto de la cocina con la ayuda de una chimenea de metal. De esa manera, humos y leñas son regulados. A pesar de los cambios que estas nuevas estructuras suponían (pues implicaban, en teoría, terminar con los fogones anteriores) y de las aparentes discordancias con el entendimiento local acerca del uso de recursos naturales, la iniciativa fue calurosamente recibida y el proyecto terminó siendo muy exitoso. Sin embargo, las razones esgrimidas por los técnicos para explicar este éxito no coinciden exactamente con la mirada local; hay varias equivocaciones en juego (Pazzarelli 2016).

Por un lado, los problemas vinculados al uso excesivo de leñas. Al preguntar si la leña es un recurso escaso en la región los huacheños responden aclarando que en los cerros donde viven no hay leña (e incluso cada vez hay menos). Sin embargo, en Huachichocana habitan simultáneamente no más de siete familias en un amplio territorio de quebradas y puna, que debería poder proveer de leña a sus, consecuentemente, siete fogones. Pero no lo hace: no hay leña. Esta afirmación, no obstante, posee desdoblamientos que se revelaban al indagar por su sentido relacional, pues ya dijimos que ni las cosas ni las personas ni cualquier otro ser, existen fuera de vínculos particulares. En este sentido, si no hay leña es porque ya no hay personas para hacerla, pues ella no existe más que como una virtualidad que debe ser actualizada (como cuando se hace carne, ver Pazzarelli 2016). Como cualquier otro modo local de hacer, la cosecha de leña sigue técnicas y procesos específicos de selección de especies y maderas que son recolectadas y transformadas en el marco de las relaciones que mantienen plantas y personas entre sí; pero, en principio, aquel no hay leña no supone que no existan maderas (biomasa) en el cerro.

Entonces ¿significaría esto que en realidad 'hay' leña y las palabras huacheñas deberían ser reconsideradas? ¿O que los técnicos no comprenden lo que las comunidades dicen? Entendemos que no hay relativizaciones posibles en la expresión no hay leña: ella describe una situación vivida por los huacheños, pero que no apunta a la 'realidad técnica' de un ambiente externo y sus motivos no se vinculan a algún tipo de sobre-explotación vinculada a prácticas tradicionales. Por el contrario, la consecuencia lógica de las prácticas locales sería que 'haya leña' y la negación anterior podría ser traducida como 'no existen las relaciones que permiten hacer leña': generalmente, porque las familias se reducen cuando las personas migran y dejan de vivir en el cerro. Nótese, entonces, un detalle: la evaluación técnica coincide con la evaluación local, pues ambos afirman que no hay leña. No hay ahí una mala interpretación, sino una "equivocación" (Viveiros de Castro 2004) pues, técnicamente, esa falta sería consecuencia de una sobre-explotación con base en prácticas tradicionales (que deben ser "adaptadas" a tecnologías eficientes), mientras que la perspectiva local señala lo contrario: es la imposibilidad de hacer leña como se hizo siempre lo que deja al paisaje de los cerros lleno de simples maderas. En la equivocación, los postulados finales son similares (no hay leña), pero las premisas iniciales se reportan a mundos distintos (criar; explotar como recurso) (Viveiros de Castro 2004:17-18) ${ }^{14}$.

El segundo problema lo constituía el exceso de humos, que según el proyecto se desdobla en dos tipos: por un lado, aquél que se sumaría al resto de las emisiones planetarias de $\mathrm{CO}_{2}$, colaborando con el efecto invernadero; por otro lado, el que inunda las cocinas, afectando los ojos y vías respiratorias. El primer tipo de humos incidiría sobre un 'bien común natural' (clima, medio ambiente), colocándose al lado de aquél que las grandes industrias con base en el carbón o en los combustibles fósiles emiten diariamente. Este argumento sugiere un tipo de distribución de culpa donde resulta difícil (por no decir, absurdo) discernir el rol de siete fogones huacheños sobre un cambio climático que apunta hacia otro tipo de responsables. Además, se articula una idea ya esbozada: la evaluación de los problemas inherentes a los conocimientos tradicionales en torno al uso de leñas, luego de la cual las comunidades son designadas como beneficiarias de una "tecnología eficiente" (ver una reflexión similar en Allen 2002: 239). El segundo tipo de exceso se articula con argumentos de medicina preventiva que sugieren regular la exposición de las personas a los humos culinarios. Sin embargo, existen diferentes opciones dentro de la arquitectura local que, sin alterar ni fogones ni leñas, regulan las concentraciones de humos: construcción de cuartos de cocina más grandes o pequeñas salidas sobre los fogones, a la manera de ventanas, entre muchas otras. Es decir, aun coincidiendo con los argumentos preventivos, existirían soluciones locales para llevarlos adelante. Para presentar el problema de otro modo, podría decirse que el proyecto pretendía reducir el peligro de ciertas 'conjunciones excesivas': entre el humo y el clima (disminuyendo el primero) y entre el humo y las personas (al extraerlo hacia afuera de las cocinas). Sin embargo, la relación de las personas con los humos es siempre un poco más complicada 
(e interesante) que la evaluación técnica, pues los fogones son frecuentemente un lugar de reunión y comunicación con 'otros'.

Un breve ejemplo: los modos del crepitar y de hablar del fuego y las formas del humo son señas que se multiplican en cada evento culinario. Las señas constituyen pequeños eventos que refieren anticipadamente a dominios que no son los de su manifestación: el cantar de algunos pájaros que anuncia la llegada de parientes, por ejemplo (Pazzarelli et al 2015; Pazzarelli 2016). Otras señas permiten anticipar las lluvias del verano, el clima y los futuros cambios en el paisaje: si el fuego humea mucho y cuesta encenderlo es seña de que las lluvias seguirán; en cambio, si enciende o arde rápido y con un humo típico es seña de que las precipitaciones disminuirán con la llegada del viento. Es solo a través del fuego que habla y del humo que hace picar los ojos que las distintas señas del paisaje se hacen presentes: los vientos de agosto, las lluvias del verano, el verdear de los cerros y la crecida de los arroyos aparecen como diferentes versiones del humo y del idioma del fuego. En otras palabras, así como los fuegos y los humos no comienzan ni terminan en la cocina (sus contornos no están allí), las relaciones del paisaje local tampoco acaban al entrar a una casa. El problema es que esto no es así con las cocinas económicas, que ocultan parcialmente el fuego en sus cajas y extraen el humo fuera de la cocina, reduciendo literal y materialmente la manifestación de ciertas señas. O como dicen las mujeres: en las cocinas económicas, el fuego no habla.

Considerando lo anterior, ¿cómo comprender entonces que el proyecto fue finalmente desarrollado y calificado de exitoso por todas las partes? Por un lado, porque se nutrió de muchas asambleas y reuniones entre comunidades y técnicos, luego de las cuales las familias tuvieron autonomía para montar las estructuras y decidir su destino. Por otro lado, las familias tomaron algunas decisiones para evitar la desaparición de fuegos y humos, que se vieron facilitadas, entre otras cosas, porque los grupos familiares siempre poseen más de una cocina. Así, las cocinas económicas se instalaron en las casas (y se usan cada vez que hay poca leña) y los fogones sobrevivieron en los puestos. Los hornos tampoco suplantaron a los antiguos, fueron construidos generalmente al lado y se utilizan para fiestas o celebraciones colectivas, cuando se necesita preparar grandes cantidades de comidas. Se produjo, entonces, una multiplicación de opciones culinarias: a los antiguos fuegueros, hornos de pan y cocinas a gas se sumaron las cocinas y hornos económicos ${ }^{15}$. Esto contradice las expectativas del proyecto sobre reducción del uso de leñas y humos, al tiempo que nos interpela sobre la aceptación local del proyecto: finalmente ¿hay coincidencias entre las perspectivas sobre cocinas, leñas y fuegos? No hay una única respuesta para lo anterior. Coincidiendo con el argumento acerca de la falta de leña las familias instalaron las cocinas; pero ese movimiento no supuso para ellas economizar humos (¡ni leñas!, como deseaba el proyecto) ni mucho menos las intromisiones que las fuerzas del paisaje hacen en los fogones de las personas.

La equivocación de las cocinas se presenta como otra "conexión parcial" (Strathern 2004): de un lado, un proyecto con financiamiento internacional que deseaba reducir humos y leñas; de otro lado, la recepción e instalación local de cocinas económicas que multiplicaron las formas culinarias y sus humos. El encuentro no resume ni sintetiza ninguno de estas 'posiciones', no hay consenso ni punto medio: ambos están conectados, pero no se cancela la diferencia que existe entre ellos. Tampoco hay malentendidos: el proyecto es en sí mismo una equivocación. Una 'equivocación productiva' o 'fértil' (cf. Holbraad et al. 2014) que sin reducirse a ninguna de sus posiciones anteriores, se abre hacia una multiplicidad culinaria y de mundos que interviene positivamente sobre la 'falta de leña'... pero sin economizar humos.

\section{Paisajes y Equivocaciones: Algunas Reflexiones Finales}

Colocar en variación al paisaje y a la imaginación conceptual que trae consigo como eje de equívocos constantes constituye menos una propuesta de trabajo con pasos definidos y más una "proposición" (Stengers 2005). Una que intente dejar florecer la mayor cantidad de relaciones posibles allí donde no sabíamos que existían, o donde no parecía posible pensarlas. De algún modo, se trata de fomentar una aptitud metodológica que haga posible encontrar lo que no se estaba buscando (Strathern 2014). En nuestro caso, debimos volver a preguntarnos por aquello que parecía evidente y hacer lugar a lo que las relaciones establecidas en el campo proponían y nos invitaban a pensar. Una perspectiva de este tipo reclama, al menos parcialmente, de aquello que la antropología contemporánea define como un "tomar en serio" las formas en que otras sociedades definen sus propias condiciones de autodeterminación ontológica (Holbraad et al. 2014; Viveiros de Castro 2010). Eso incluye, claro, a todos los seres que piensan y se mueven junto con las personas. De los breves recorridos previos se desprenden algunas consideraciones a tener en cuenta. 
Por un lado, hemos sugerimos que las formas prácticas de vincularse al paisaje reclaman de una serie de relaciones que, sin negar posibles conexiones de carácter occidental u histórico-moderno, se acomodan mejor a lo que definimos como modulaciones de una memoria fértil. Por otro lado, como expresión más concreta de estas relaciones, explicitamos el lugar de las cocinas y sus humos como transformaciones de otros vientos y movimientos de aire, en articulación con un paisaje repleto de seres y fuerzas que se involucran en la vida cotidiana. En ambos casos, sugerimos que estos vínculos pueden absorber y digerir relaciones 'ajenas' (prácticas antropológicas y arqueológicas, turismo, argumentos ambientales, cocinas), para transformarlas en otras cosas. Entendemos que debemos tomar en serio los distintos alcances de estas relaciones, sin sobrecodificarlas a priori como si fuesen manifestaciones colonizadas de un pensamiento prístino anterior que solo nosotros conseguiríamos ver plenamente. En otras palabras, deberíamos llevar hasta las últimas consecuencias los modos en que los seres del paisaje se encargan de conectarse con aquello que viene de fuera, con la posibilidad de transformarlo creativa y positivamente.

Esta perspectiva de ninguna manera supone desactivar la lectura de los frecuentes conflictos y violencias que relaciones de este tipo pueden suponer. No estamos sugiriendo que la capacidad huacheña, y de los seres de su paisaje, por absorber y transformar relaciones y categorías foráneas posea resultados siempre positivos (como bien muestran Spedding y Arnold 2009). Mucho menos que algo así sea pensable en otras situaciones, donde la maquinaria estatal y del mercado se ocupa simplemente de aplastar las relaciones que no le convienen (ver, p.ej. De la Cadena 2015). Lo que deseamos resaltar es que el compromiso con nuestro campo reclama considerar simétricamente todas las formas creativas (y no solo algunas) con las que las personas consiguen componer y habitar su mundo. Esto incluye aquellas situaciones que suponen experiencias vitales momentánea, o potencialmente, fértiles y evaluadas locamente como positivas. Ninguna de estas relaciones deberían ser reducidas a esquemas ajustados a nuestros principios de contradicción (o, peor, a nuestros juicios acerca de lo que los otros deberían estar haciendo). Ello impediría imaginar, por ejemplo, la posibilidad de que una cueva pueda ser presentada como un antigal potente y criador y como un sitio arqueológico turístico, o un cerro como un ser pensante y un espacio capaz de ser mensurado, casi al mismo tiempo. No porque los proyectos de turismo (o de desarrollo o de investigación antropológica) sean naturalmente deseables, positivos o el mejor destino para una comunidad indígena; sino porque a la hora de enfrentarse a ellos y pensarlos, los huacheños lo hacen de un modo diferente al nuestro. $\mathrm{Al}$ respecto, resulta pertinente la reflexión de Viveiros de Castro (2016) sobre el desencuentro entre los Yanomami y los blancos al discutir los usos y demarcaciones del territorio: los primeros han aprendido las 'reglas de juego de los blancos' y no dudan de la importancia de la demarcación del territorio frente al Estado ${ }^{16}$. No porque esa demarcación sea naturalmente interesante o deseable, sino porque se trata de una condición (injustamente obligatoria) para que la tierra continúe en manos indígenas y por lo tanto, podríamos decir, 'viva'.

Negar a priori, entonces, la posibilidad de que el paisaje y sus relaciones puedan ser enunciadas y practicadas en muchos términos diferentes, inclusive algunos parcialmente (y momentáneamente) conectados a nuestras ideas de representación o recurso, reduce drásticamente nuestras posibilidades de comprensión como antropólogos (y también como asistentes de técnicos, si fuera el caso). En otras palabras, se trata de considerar seriamente y explicitar los modos en que los huacheños ensanchan creativamente el espacio disponible para pensar las relaciones 'con' los cerros al mismo tiempo que sus relaciones 'con' los antropólogos (y otros) 'sobre' los cerros. Debemos reconocer aquella capacidad creativa que establece relaciones entre mundos, haciendo de la convivencia de las diferencias algo fértil, al menos momentáneamente (en los sentidos que lo ch'ixi tendría, Rivera Cusicanqui 2010). Si no lo hiciéramos, caeríamos en aquellas formulaciones que dudan de la seriedad ontológica de las relaciones con los antigales desde el momento en que una comunidad acepta el trabajo arqueológico; o en aquellas que relativizan las implicaciones mutuas con los seres del paisaje desde el instante en que una familia decide transformar una de sus cocinas.

Todo se resume en la reflexión que Goldman (2016) realiza sobre el oficio del antropólogo, que debe decidir a cada instante si va a apoyarse sobre la "debilidad" o sobre la "fuerza" de las personas con las que trabaja. Aprender de la fuerza de los huacheños supone, en nuestro caso, aprender de aquella capacidad para hacer florecer relaciones incluso allíen donde parecería que nada puede crecer, e independientemente de si nuestra moral académica opina que son interesantes o potencialmente fértiles los vínculos entre comunidades y el Estado, ONGs o emprendimientos privados.

En segundo lugar, algo que resulta fundamental para este trabajo, es la necesidad de considerar también a las propias relaciones locales con los seres del paisaje como equívocas. Si algo debemos 
aprender de nuestro trabajo de campo es que los conflictos que median las conversaciones sobre el paisaje no solo se revelan cuando un turista, un técnico o un antropólogo se presentan en la comunidad. Las equivocaciones ocurren incluso hacia el interior de las relaciones de crianza cuando, por ejemplo, entran en disputa las miradas de las personas y de los cerros o cuevas sobre la "realidad" de un evento (para usar la expresión de Spedding 1992: 329)17. Aquí reside, creemos, la mejor de las enseñanzas huacheñas: el paisaje no es 'objeto' de disputa (entre locales y foráneos que no se comprenden mutuamente) sino un conjunto de seres y fuerzas que se implican activamente en las equivocaciones. Y no lo hace solo porque está 'vivo', sino porque está vivo en relación a alguien. En estas relaciones, lo que el mundo puede o no puede ser está siempre siendo contestado en prácticas específicas que intentan modular las formas en que las relaciones son objetivadas. Nadie puede saber con certeza si una relación va a desencadenar otras positivas. Las challas, los pagos, la interpretación de las señas y la regulación de los humos constituyen momentos en que se modulan las formas en las que personas y otros seres perciben y fabrican sus mundos junto a las conexiones que los articulan. Este antecedente relacional es el que permite pensar luego en la posibilidad de una conexión con el mundo moderno y sus formas. En otras palabras, la equivocación con arqueólogos y técnicos puede llegar a ser productiva solo porque una variación positiva de ella ya existe en la relacionalidad local.

Considerado esto, entonces, que el paisaje sea pensado como un conjunto relacional que está 'vivo' no es la respuesta definitiva a ninguna de nuestras preguntas. Es solo un inicio para comenzar a mapear qué tipo de 'vida' o 'relación' reclaman los cerros en Huachichocana y cómo son moduladas a cada paso; solo entonces, preguntarse si acaso eso tiene algo que ver con nuestras propias ideas de fabricar conexiones con 'otros'. Algunos pasos hemos dado en este sentido. Habitar y controlar equivocaciones parece ser la primera de las enseñanzas que puede proporcionarnos una mirada sobre el paisaje huacheño: enfatizar la posibilidad de habitar parcialmente mundos múltiples, sin pretender erosionar las diferencias entre ellos. Pues finalmente, la inquietud final de este texto sería la de contornear las formas en que las relaciones de un paisaje se presentan como irreductibles a 'un' tipo de mundo, comprender que reclaman de más de uno para ser comprendidas y aceptar que la diferencia que ellas suponen hace imposible que podamos pensarlas más que bajo la forma de conexiones parciales.

Agradecimientos: A las familias y personas que integran Huachichocana. Gracias a ellos, a su tierra y a sus cerros por albergarnos y permitirnos afectuosamente conectar con su mundo.

\section{Referencias Citadas}

\begin{abstract}
Allen, C. 2002. The Hold Life Has: Coca and Cultural Identity in an Andean Community. Smithsonian Institution Press, Washington y London.
\end{abstract}

Arnold, D. 2009. Cartografías de la memoria: hacia un paradigma más dinámico y viviente del espacio. Cuadernos FHyCS-Universidad Nacional de Jujuy 36:205-246.

Arnold, D. (coord.), D. Jiménez Aruquipa y J. de D. Yapita 1992. Hacia un Orden Andino de las Cosas: Tres Pistas de los Andes Meridionales. ILCA, La Paz.

Bastien, J. 1996. La Montaña del Cóndor. Metáfora y Ritual en un Ayllu Andino. Hisbol, La Paz.

Bouysse-Cassagne, T. 2004. El sol de adentro: wakas y santos en las minas de Charcas y en el lago Titicaca (siglos XV a XVIII). Boletín de Arqueología PUCP 8:59-97.

Bouysse-Cassagne, T. y O. Harris 1987. Pacha: en torno al pensamiento aymara. En: Tres Reflexiones sobre el Pensamiento Andino, editado por T. Bouysse-Cassagne, O. Harris, V. Cereceda y T. Platt, pp. 11-60. Hisbol, La Paz.

Bugallo L. 2008. Marcas del espacio andino de la Puna de Jujuy: un territorio señalado por rituales y producciones. En Paisaje, Espacio y Territorio. Reelaboraciones Simbólicas y
Reconstrucciones Identitarias en América Latina, editado por N. Ellison y M. Martínez Mauri, pp. 69-88. Abya-Yala, Quito.

Bugallo, L. y J. Tomasi 2012. Crianzas mutuas. El trato a los animales desde las concepciones de los pastores puneños (Jujuy, Argentina). Revista Española de Antropología Americana 42:205-224.

Bugallo, L. y M. Vilca 2011. Cuidando el ánimu: salud y enfermedad en el mundo andino (puna y quebrada de jujuy, argentina). Nuevo Mundo, Mundos Nuevos, http://nuevomundo.revues.org/61781 (último acceso 28/12/17).

Cruz, P. 2012. El mundo se explica al andar. Consideraciones en torno a la sacralización del paisaje en los Andes del sur de Bolivia (Potosí, Chuquisaca). Indiana 29:221-251.

Cruz de Tolaba A., B. Tolaba, C. Mamani, C.G. Tolaba, F. Cruz, F. Tolaba, G.D. Mamani, G. Martínez de Cruz, I. Cruz, I. Zambrano, J. Casimiro, J.C. Martínez, J. Barrionuevo, J. Tolaba, L. Mamani, M.I. Cruz, M. Cruz, M. Tolaba, P. Mamani, P. Barrionuevo, P. Mamani, S.J. Cruz, T. Cruz, U. Tolaba, V. Tolaba, V. Mamani, V. Martínez e Y. Mamani 2015. De Pircas, Cardones, Rastrojos, Chivos y Cuevas. Historia de la Comunidad Aborigen de Huachichocana. Edición de la Comunidad de Huachichocana, La Plata. 
De la Cadena, M. 2015. Earth Beings. Ecologies of Practices across Andean Worlds. Duke University Press, Duke y Londres.

Favret-Saada, J. 1990. Être Affecté. Gradhiva. Revue d'Histoireetd'Archives del'Anthropologie 8:3-9.

Goldman, M. 2016. Prólogo. En Vivir Sabroso. Luchas y Movimientos Afroatrateños, en Bojayá, Chocó, Colombia, editado por N. Quiceno Toro, pp. 1-7. Editorial Universidad del Rosario, Bogotá.

Gose, P. 2001 Aguas mortíferas y Cerros Hambrientos. Rito Agrario y Formación de Clases en un Pueblo Andino. Mama Huaco, La Paz.

Gow, P. 1995. Land, people and paper in Western Amazonia. En The Anthropology of Landscape: Perspectives of Place and Space, editado por E. Hirsch y O’Hanlon, pp. 43-62. Oxford University Press, Oxford.

Haber, A. 2007. Arqueología de uywaña: un ensayo rizomático. En Producción y Circulación Prehispánicos de Bienes en el Sur Andino, editado por A. Nielsen, C. Rivolta, V. Seldes, M. Vázquez y P. Mercolli, pp. 13-36. Editorial Brujas, Córdoba.

Holbraad, M., M. APedersen y E.Viveiros de Castro 2014. The politics of ontology: Anthropological positions. Fieldsights Theorizing the Contemporary, Cultural Anthropology Online http://culanth.org/fieldsights/462-the-politics-of-ontologyanthropological-positions (últimoacceso 28/12/17).

Kopenawa, D. y B. Albert (eds.) 2016. A Queda do Céu. Palavras de um Xamã Yanomami. Companhia das Letras, São Paulo.

Latour, B. 2007 [1991]. Nunca Fuimos Modernos. Ensayo de Antropología Simétrica. Siglo Veintiuno Editores, Buenos Aires.

Lema, V. 2013. Crianza mutua: una gramática de la sociabilidad andina. Actas de la X Reunión de Antropología del Mercosur. CD Room.

Lema, V. 2014a. Criar y ser criados por las plantas y sus espacios en los Andes septentrionales de Argentina. En Espacialidades Altoandinas. Avances de Investigación desde el Noroeste argentino, editado por A. Benedetti y J. Tomasi, pp. 301-338. FFyL-UBA, Buenos Aires.

Lema, V. 2014b. Hacia una cartografía de la crianza: domesticidad y domesticación en comunidades andinas. Espaço Amerindio 8:59-82.

Lema, V. y F. Pazzarelli 2015. Memoria fértil. Crianza de la historia en Huachichocana. Nuevo Mundo Mundos Nuevos http:// nuevomundo.revues.org/67976; DOI: 10.4000/nuevomundo. 67976 (último acceso 28/12/17).

Lema, V. y F. Pazzarelli 2018. Las formas de la historia. Equívocos, saberes y memorias en los cerros jujeños. $R @ U$. Revista de Antropologia da UFSCar. En prensa.

Martínez, G. 1983. Los dioses de los cerros en los Andes. Journal de la Société des Américanistes 69:85-115.

Martínez, G. 1989. Espacio y Pensamiento I. Andes Meridionales. Hisbol, La Paz.

Molinié, A. 1997. Buscando una historicidad andina: Una propuesta antropológica y una memoria hecha rito. En Arqueología, Antropología e Historia en los Andes: Homenaje a María Rostworowski, pp. 691-708. Instituto de Estudios Peruanos, Lima.

Pazzarelli, F. 2010. La importancia de hervir la sopa. Mujeres y técnicas culinarias en los Andes. Antípoda 10:157-181.

Pazzarelli, F. 2014. Un queso entre otros. Sueros, familias y relaciones en los cerros jujeños. Revista Colombiana de Antropología 50:95-118.
Pazzarelli, F. 2016. La equivocación de las cocinas. Humos, humores y otros excesos en los Andes Meridionales. Revista de Antropologia 59:49-72.

Pazzarelli, F. 2017. A sorte da carne. Topologia animal nos Andes Meridionais. Horizontes Antropológicos 23:129-149.

Pazzarelli, F. y V. Lema 2015. Masticar-digerir-deshidratar: lógicas de la transformación en challas de arrieros. Ponencia presentada en las Primeras Jornadas sobre el Altiplano Sur: Miradas Disciplinares, pp. 33-34. Tilcara, Jujuy.

Pazzarelli, F., B. Marconetto y M. Bussi 2015. Le vent qui seche les chèvres. Quelques réflexions autour de la cuisine, de la nourriture et des 'phénomènes météorologiques' dans les Andes meridionales". En L'Alimentation et le temps qu'il fait, editado por K. Becker, V. Moriniaux y M. Tabeaud, pp. 95-104. Éditions Hermann, Paris.

PNUD (Programa de las Naciones Unidas para el Desarrollo) 2009. Programa de Pequeñas Donaciones en Argentina. Buenos Aires.

Quiroga Mendiola, M. 2012. Llueve sobre mojado... Trashumancia conceptual frente al pastoralismo alto andino. En La Desigualdad ¿del Desarrollo? Controversias y Disyuntivas en el Desarrollo Rural del Norte Argentino, editado por M. Manzanal y M. Ponce. CICCUS, Buenos Aires.

Rivera Cusicanqui, S. 2010. Ch'ixinakaxutxiwa. Una reflexión sobre Prácticas y Discursos Descolonizadores. Tinta Limón editores, Buenos Aires.

Spedding, A. 1992. Almas, anchanchus y alaridos en la noche: el paisaje vivificado de un valle yungueño. En Economía, Etnicidad y Simbolismo en los Andes, editado por S. Arze, pp. 299-330. IFEA, Lima.

Spedding, A. y D. Arnold 2009. La ritualidad en el Altiplano del pasado y de hoy en un marco identitario. En ¿Indígenas u Obreros? La Construcción Política de Identidades en el Altiplano Boliviano, editado por D. Arnold, pp. 311-351. UNIR, La Paz.

Stengers, I. 2005. The cosmopolitical proposal. En Making Things Public. Atmospheres of Democracy, editado por B. Latour y P. Weibel, pp. 994-1003. MIT Press, Cambridge.

Strathern, M. 2004. Partial Connections. AltaMira, New York.

Strathern, M. 2014 [1999]. O efeito etnográfico. En O Efeito Etnográfico, editado por M. Strathern, pp. 345-405. CosacNaify, São Paulo.

Tomasi, J. 2011. Geografías del Pastoreo. Territorios, Movilidades y Espacio Doméstico en Susques (Provincia de Jujuy). Tesis doctoral inédita, Facultad de Filosofía y Letras, Universidad de Buenos Aires, Buenos Aires.

Vilca, M. 2009. Más allá del 'paisaje'. El espacio de la puna y quebrada de Jujuy: ¿comensal, anfitrión, interlocutor? Cuadernos FHyCS-Universidad Nacional de Jujuy 36:245259.

Vilca, M. 2012. El Diablo por la cocina. Muertos y diablos en la vida cotidiana del norte jujeño Estudios sociales del NOA, nueva serie 12:45-58.

Viveiros de Castro, E. 2004. Perspectival Anthropology and the Method of Controlled Equivocation. Tipiti: Journal of the Society for the Anthropology of Lowland South America 2(1): 3-22.

Viveiros de Castro, E. 2010. Metafísicas Caníbales. Líneas de Antropología Postestructural. Katz Editores, Buenos Aires. 
Viveiros de Castro, E. 2016. O recado da mata. En A Queda do Céu. Palavras de um Xamã Yanomami, editado por D.
Kopenawa y B. Albert, pp. 11-41. Companhia das Letras, São Paulo.

\section{Notas}

1 Empleamos cursivas para referirnos a expresiones nativas, comillas dobles para citas de otros autores y comillas simples para relativizar palabras o expresiones de nuestra autoría.

2 Desde 2010, nuestro trabajo se desarrolla a través de proyectos de corte etnográfico y arqueológico (estos últimos a cargo de una de las autoras, V.L.). Las estancias de trabajo de campo se multiplicaron a lo largo de estos años, desde visitas sociales hasta estancias de varios meses de duración. Entre las tareas que nos ocuparon se cuentan actividades de tipo colaborativas que, entre otras cosas, terminaron en la producción conjunta de un libro sobre la historia de la comunidad (Cruz de Tolaba et al. 2015).

3 A título comparativo, es interesante la reflexión de Gow (1995) para Amazonia occidental, donde el paisaje no encerraría ninguna posibilidad de "representación" ni posibilidad de punto de vista externo que pueda totalizarlo; el antropólogo debe involucrarse activamente (y hasta devenir pariente) para comprender esas relaciones.

4 “... lo ch'ixi conjuga el mundo indio con su opuesto, sin mezclarse nunca con él (...) La hibridez asume la posibilidad de que de la mezcla de dos diferentes, pueda salir un tercero completamente nuevo (...) La noción de ch'ixi (...) plantea la coexistencia en paralelo de múltiples diferencias culturales que no se funden, sino que antagonizan o complementan" (Rivera Cusicanqui 2010: 70).

5 En Huachichocana existen estaciones de arte rupestres, sitios a cielo abierto y sitios en cuevas que atestiguan una ocupación de larga duración, desde el 10.000 a.p. hasta momentos post-conquista. Esta destacada riqueza hizo que la arqueología local sea conocida tanto en el ámbito académico como a nivel turístico local, llegando usualmente visitantes desde el cercano pueblo de Purmamarca, interesados por las cuevas y sus pinturas.

6 La tijtincha es una comida preparada a base de carne seca y carne fresca (con especial participación de las cabezas de animales criados) y mazorcas secas de maíz que se hierven durante muchas horas.

7 O como cuando un cerro ancestral pasa a tomar el nombre de un empresario u antropólogo (como muestra Cruz 2012)

8 Lo que supone que otras personas puedan comenzar a formar parte de la memoria local al involucrarse en la red, independientemente (al menos, en principio) de que tengan relaciones 'ancestrales' con el lugar. Las reflexiones de Bastien sobre el ayllu de Kaata son interesantes al respecto: "El ayllu se refiere también a la gente que vive en el mismo territorio (llahta) y que alimenta a los santuarios dedicados a la tierra de ese territorio. Los migrantes peruanos, por ejemplo, se trasladaron a Kaata alrededor del año 1920, se convirtieron en miembros de los grupos descendientes de ese nivel al alimentar a los antepasados de los kaateños nativos (...) Al vivir en la montaña y alimentar ritualmente a sus santuarios, la gente se convertía en una parte del ayllu de la montaña, sin importar de dónde venían" (Bastien 1996: 25). La cita anterior sugiere que la pertenencia al ayllu (y por tanto, la memoria) se define (al menos parcialmente) en el territorio práctico de la alimentación a los cerros (ver también Lema y Pazzarelli 2015).

9 Ello también quedó manifestado durante la escritura conjunta de un libro sobre la memoria del lugar (Cruz de Tolaba et al 2015). El equívoco entre ambos 'productos' (nuestro trabajo académico y el libro realizado a nombre de la comunidad) es explorado en Lema y Pazzarelli 2018.

10 En el mismo sentido, los proyectos de turismo que los huacheños anhelan y reclaman están aún en los papeles; así también la creación de un museo local para la exhibición de los restos arqueológicos que aportaron las excavaciones (realizadas desde la década de 1970).

11 Son numerosos los relatos de personas enviciadas con la búsqueda de riquezas, tentadas por las cuevas: algunas volvieron para contarlo (gracias, por ejemplo, a la intercesión de la Virgen), otras aparecieron en lugares lejanos, otros fueron loqueados, otros desaparecieron. En todos los casos, los involucrados no siguieron los protocolos recomendados para relacionarse con las cuevas.

12 Hasta el momento no hemos abordado sistemáticamente la articulación del dinero en la relacionalidad local. Podríamos sugerir, no obstante, que los huacheños se encuentran interesados en que las potencias 'fértiles' del dinero no se escapen en dirección a aquellos que no son criadores del lugar (sean los arqueólogos del pasado que han vendido libros con su historia o los guías de turismo que vienen de afuera y cobran por visitar las cuevas)

13 Estos proyectos fueron desarrollados en muchas otras comunidades de diferentes provincias de la región noroeste de la Argentina y de la región de los Andes meridionales. No pretendemos generalizar nuestro análisis, pero creemos que la situación aquí presentada puede contener algunos puntos de interés comunes a otras experiencias.

14 El problema de la leña sería tal vez análogo al de "sobrepastoreo" de los pastores puneños, sobre el cual trabajan distintos programas de desarrollo (Quiroga Mendiola 2012).

15 Esta multiplicación tiene otras relaciones que se vinculan con la organización culinaria diferencial de los distintos espacios habitados: casas y puestos albergan diferentes cotidianeidades que se refleja en la disponibilidad de objetos y estructuras.

16 ¿A su pueblo le gustaría recibir informaciones sobre cómo cultivar la tierra? / No. Lo que deseo obtener es la demarcación de nuestro territorio. Diálogo entre el general BaymaDeny y DaviKopenawa, durante la audiencia con el presidente José Sarney, 19 de abril de 1989" (Epígrafe al capítulo 17 de Kopenawa y Albert 2016: 376 -nuestra traducción). Este fragmento es retomado por Viveiros de Castro (2016: 35-36) en su prefacio al mismo libro, para argumentar sobre la "diferencia indígena" implicada en la respuesta del chamán; diferencia que reposa en un equívoco que solo una de las partes se encuentra interesada en explicitar.

17 Llevado hasta sus últimas consecuencias, el equívoco debería ser considerado incluso como interno al ser, a la propia existencia siempre e inevitablemente dividida (Viveiros de Castro 2016: 31). 